\title{
Avaliação do vigor de sementes de girassol por meio de análise de imagens de plântulas
}

\author{
Sunflower seed vigor evaluation by seedling image analyze
}

\section{Cartiane Rubshirley Macêdo da Rocha ${ }^{\mathrm{I}}$ Vanessa Neumann Silva ${ }^{\mathrm{II}}$ Silvio Moure Cicero ${ }^{\mathrm{I}}$}

\section{RESUMO}

O trabalho teve como objetivo avaliar a eficiencia do sistema computadorizado de análise de imagens de plântulas Seed Vigor Imaging System (SVIS ${ }^{\circledR}$ ), em comparação com os outros testes recomendados para determinação do vigor de sementes de girassol. Foram utilizados dez lotes de sementes, cultivar Catissol 01, os quais foram avaliados quanto ao potencial fisiológico por meio dos testes de germinação, envelhecimento acelerado, emergência de plântulas em areia e em campo e análise de imagens de plântulas com o sistema $S V I S^{\circledR}$, aos 0 e 4 meses de armazenamento. $O$ delineamento experimental utilizado foi inteiramente casualizado para os testes realizados em laboratório e blocos ao acaso para o teste de emergência de plântulas em campo; em ambos os casos, foram utilizadas 4 repetições. Os dados foram submetidos à análise de variância e as médias comparadas pelo teste de Scott Knott a 5\% de probabilidade. Verificou-se que a análise computadorizada de imagens de plântulas com SVIS ${ }^{\circledR}$ é eficiente para avaliação do vigor de sementes de girassol.

Palavras-chave: Helianthus annus L., potencial fisiológico, SVIS ${ }^{\circledR}$

\section{ABSTRACT}

This study aimed to evaluate the efficiency of computerized image analysis of seedling Seed Vigor Imaging System $\left(S V I S^{\mathbb{R}}\right)$ compared with the other recommended tests to determine vigor in sunflower seeds. It was used ten seed lots of cultivar Catissol 01.The physiological potential was assessed using tests of germination, accelerated aging, seedling emergence and seedling image analyze with SVIS ${ }^{\circledR}$ at 0 and 4 months after storage. The experimental design was completely randomized for the laboratory tests and randomized blocs to seedling emergence on the field; in both cases it was used four replications. The data were submitted to variance analyze and mean comparisons at Scott
Knott test $(P<0,01)$. Seedling image analyze with SVIS ${ }^{\circledR}$ is efficient to access sunflower vigor seeds.

Key words: Helianthus annus L., physiological potential, SVIS ${ }^{\circledR}$.

\section{INTRODUÇÃO}

A cultura do girassol (Helianthus annuus L.), juntamente com a soja e a canola, possui importância significativa na economia mundial, apresentando-se como uma das três maiores produtoras de óleo do mundo (FAGUNDES, 2009). Nesse contexto, é essencial que os produtores disponham de sementes de alta qualidade para o adequado estabelecimento de plantios comerciais, possibilitando um rápido e adequado desenvolvimento das plantas, condições imprescindíveis para a obtenção de lavouras com elevadas produtividades.

A disponibilidade de testes eficientes para análise é fundamental para o controle de qualidade na produção de sementes. O potencial fisiológico de sementes pode ser avaliado por meio dos testes de germinação e de vigor, contudo, como o teste de germinação é realizado em condições ótimas, pode superestimar o potencial de lotes de sementes.

Para auxiliar na obtenção de informações a respeito do potencial de desempenho de lotes de sementes em condições variadas de ambiente, surgiram os testes de vigor. Segundo TEKRONY (2003), vigor de sementes é uma característica complexa, comparada

'Departamento de Produção Vegetal, Escola Superior de Agricultura 'Luiz de Queiroz', Universidade de São Paulo, Piracicaba, SP, Brasil. IUniversidade Federal do Pampa, Campus Itaqui, 97650-000, Itaqui, RS, Brasil. E-mail: vanessaneumann@unipampa.edu.br. Autor para correspondência. 
à germinação, e proporciona informação adicional para auxiliar na diferenciação de lotes de sementes com níveis aceitáveis de germinação.

Considerando que a manifestação do vigor de sementes resulta da interação das características que determinam seu potencial para uma rápida e uniforme emergência de plântulas e o estabelescimento de plantas em condições variadas de ambiente (BAALBAKI et al., 2009), o uso de um único teste para avaliação do vigor pode não ser uma maneira eficiente de obter informações a respeito do potencial de desempenho de lotes de sementes.

$\mathrm{Na}$ análise do vigor de sementes, buscase o uso de procedimentos que sejam de baixo custo, de fácil execução e que permitam a obtenção de resultados confiáveis em curto espaço de tempo. Embora alguns testes de vigor produzam resultados confiáveis, sempre há espaço para a continuidade da pesquisa e aprimoramento de metodologia ou inclusão de alternativas inovadoras (MARCOS FILHO et al., 2009), como as análises automatizadas de imagens de sementes e de plântulas.

A literatura tem documentado vários estudos envolvendo a análise de imagens em Tecnologia de Sementes, visando a estabelecer a relação com o vigor de sementes (MARCOS FILHO et al., 2009). Recentemente, vários trabalhos de pesquisa indicam eficiência do uso de análise computadorizada de plântulas com o sistema SVIS ${ }^{\circledR}$ na avaliação do vigor de sementes de berinjela (SILVA \& CICERO, 2014), trigo (SILVA et al., 2012a), milho doce (ALVARENGA et al., 2012), crotalária (SILVA et al., 2012b), pepino (CHIQUITO et al., 2012), entre outras.

$\mathrm{O}$ procedimento para avaliação do vigor de sementes por meio do SVIS $^{\circledR}$ é simples e os resultados da análise de imagens para uma amostra de 50 sementes podem ser obtidos dentro de dois a três minutos, além de propiciar menor subjetividade da análise pelo analista, aumentando a confiabilidade dos dados para fins de comparação (GOMES JUNIOR et al., 2009).

Dessa forma, o objetivo deste trabalho foi avaliar a eficiência do sistema computadorizado de análise de imagens de plântulas Seed Vigor Imaging System $\left(\mathrm{SVIS}^{\circledR}\right.$ ), em comparação com outros testes recomendados para determinação do vigor de sementes de girassol.

\section{MATERIAL E MÉTODOS}

A pesquisa foi realizada nos Laboratórios de Análise de Sementes e Análise de Imagens e no campo didático experimental do Departamento de
Produção Vegetal, pertencentes à Universidade de São Paulo, Escola Superior de Agricultura "Luiz de Queiroz", em Piracicaba, SP. Foram utilizadas sementes de girassol, cultivar Catissol 01, sem tratamento químico, representadas por 10 lotes. As sementes foram avaliadas quanto ao grau de umidade, germinação e vigor assim que foram adquiridas e após 4 meses de armazenamento. As sementes foram armazenadas em sacos de papel Kraft em sala de ambiente controlado $\left(20^{\circ} \mathrm{C}\right.$ e $45 / 50 \%$ UR ar). Os testes utilizados para avaliação das sementes foram executados conforme metodologia descrita na sequência.

Determinação do grau de umidade realizada pelo método da estufa $\left(105^{\circ} \mathrm{C} \pm 3^{\circ} \mathrm{C}\right)$, durante 24 horas com resultados expressos em porcentagem (base úmida) por lote (BRASIL, 2009). Germinação - preliminarmente foi realizado teste para verificar se os lotes apresentavam sementes dormentes. Como não houve dormência, procedeu-se ao teste padrão de germinação, com quatro repetições de 50 sementes por lote, as quais foram semeadas em rolos de papel de germinação umedecidos com quantidade de água equivalente a 2,5 vezes a massa do papel não hidratado e mantidas a $25^{\circ} \mathrm{C}$ em germinador. As avaliações foram realizadas de acordo com os critérios estabelecidos nas Regras para Análise de Sementes (BRASIL, 2009), com resultados expressos em porcentagem de plântulas normais. Envelhecimento acelerado - uma fina camada de sementes foi distribuída sobre tela metálica acoplada a caixas de plástico transparente $(11 \times 11 \times 3,5 \mathrm{~cm})$, contendo $40 \mathrm{ml}$ de água no interior. As caixas foram mantidas em câmara do tipo Biochemical Oxygen Demand, a $41^{\circ} \mathrm{C}$, durante 72 horas (MARCOS FILHO, 1999). Após esse período, foi determinado o grau de umidade das sementes e realizado o teste de germinação segundo as Regras para Análise de Sementes (BRASIL, 2009), com avaliação realizada aos quatro dias após a semeadura e os resultados expressos em porcentagem. Emergência de plântulas em areia - quatro repetições de 50 sementes por lote foram distribuídas na superfície de uma camada de $5 \mathrm{~cm}$ de areia, colocada em caixas plásticas $(32 \times 28 \times 10 \mathrm{~cm})$, cobertas com uma camada de $2 \mathrm{~cm}$ de areia; o umedecimento do substrato foi efetuado com quantidade de água correspondente a $60 \%$ da capacidade de retenção hídrica do substrato (BRASIL, 2009). As caixas foram expostas à temperatura ambiente durante 14 dias e, após esse período, foi determinada a porcentagem média de emergência de plântulas para cada lote. A velocidade de emergência de plântulas foi obtida registrando- 
se diariamente o número de plântulas emersas até o décimo quarto dia após a semeadura. O índice de velocidade de emergência foi calculado de acordo com a fórmula proposta por MAGUIRE (1962). Emergência de plântulas em campo - conduzido com quatro repetições, representadas por uma linha de $4 \mathrm{~m}$ de comprimento, onde foram distribuídas 50 sementes com espaçamento entre si de $8 \mathrm{~cm}$ e entre linhas de $50 \mathrm{~cm}$, em sulcos de $7 \mathrm{~cm}$ de profundidade, cobertos com $3 \mathrm{~cm}$ de terra. A contagem das plântulas emersas foi realizada no décimo quarto dia após a semeadura, com os resultados expressos em porcentagem. Análise de imagens de plântulas com o auxílio do sistema computacional Seed Vigor Imaging System $\left(S V I S^{\circledR}\right)$ - inicialmente, foram realizadas avaliações preliminares para verificar o tamanho de plântulas mais adequado para análise, visando à obtenção rápida e eficiente destas para a aquisição das imagens e avaliação. Foram testados os períodos de 2,3 e 4 dias de exposição das sementes a $25^{\circ} \mathrm{C}$, em rolos de papel de germinação, em presença e ausência de luz, sendo definido o período de três dias de idade em ausência de luz como a melhor condição para obtenção de plântulas. Para obtenção das imagens, foram utilizadas quatro repetições de 25 sementes, semeadas em rolo de papel umedecido com a quantidade de água equivalente a 2,5 vezes a massa do papel não hidratado e mantidas em germinador no escuro a $25^{\circ} \mathrm{C}$ por três dias. Em seguida, as plântulas foram transferidas para uma folha de cartolina com coloração preta e dimensões de $30 \mathrm{~cm} \times 22 \mathrm{~cm}$ e levadas a um scaner, modelo HP Scanjet 2004, montado de maneira invertida no interior de uma caixa de alumínio com $60 \mathrm{~cm} \times 50$ $\mathrm{cm} \times 12 \mathrm{~cm}$ para obtenção de imagens de $100 \mathrm{dpi}$. Posteriormente, as imagens foram analisadas com o uso do software SVIS $^{\circledR}$. Nessa análise, o eixo raiz primária-hipocótilo de cada plântula foi marcado em vermelho. No entanto, foram necessárias correções manuais, realizadas com auxílio do 'mouse', para partes das plântulas que não foram marcadas automaticamente. Após a análise e avaliação de cada plântula, o software gerou automaticamente valores numéricos referentes a um índice de vigor (valores de 0 a 1000, diretamente proporcionais ao vigor), de crescimento e de uniformidade de crescimento das plântulas (ambos também de 0 a 1000). Depois de computados esses valores, o cursor foi posicionado sobre cada plântula, indicando automaticamente o valor correspondente ao comprimento da plântula em 'pixels'. Após a tabulação, os resultados foram transformados para $\mathrm{cm}$, considerando-se que um 'pixel'corresponde a $0,0254 \mathrm{~cm}$.
O delineamento experimental utilizado foi inteiramente casualizado para os testes realizados em laboratório e, para o teste de emergência de plântulas em campo, foi adotado o delineamento blocos ao acaso, em ambos os casos foram utilizadas quatro repetições. Os dados foram submetidos à análise de variância e as médias comparadas pelo teste de Scott Knott a 5\% de probabilidade.

\section{RESULTADOS E DISCUSSÃO}

Os resultados revelaram pequena diferença entre os lotes em relação ao grau de umidade das sementes, que variou de 9,5 a $10,6 \%$ e 7,4 a $8,1 \%$ na primeira e segunda época de avaliações, respectivamente. Assim, esse parâmetro não afetou o comportamento das sementes durante os testes realizados, visto que as variações estavam dentro de limites toleráveis, ou seja, 2 a 3 pontos percentuais (MARCOS FILHO, 1999).

$\mathrm{Na}$ caracterização inicial dos lotes, na primeira época, foi possível observar diferenças de desempenho entre os lotes com todos os testes utilizados. $O$ teste de germinação propiciou a identificação de diferenças entre os lotes, com a formação de três níveis distintos, sendo os lotes 5,9 e 10 de maior desempenho, os lotes 4 e 7 de pior desempenho e o restante de comportamento intermediário (Tabela 1). Embora a literatura relate que, se houver diferença no potencial germinativo, não há necessidade de avaliar o vigor, é interessante ressaltar que o potencial fisiológico de sementes é compreendido como a soma da capacidade germinativa e do vigor. Portanto, o uso de testes de vigor, que buscam respostas complementares às fornecidas pelo teste de germinação, pode possibilitar a obtenção de informações mais consistentes sobre o potencial fisiológico dos lotes de sementes (OHLSON et al., 2010).

Nos demais testes utilizados, observou-se a mesma tendência de ranqueamento dos lotes, com destaque, na maioria dos casos, para a superioridade dos lotes 9, classificado como intermediário pelo envelhecimento acelerado e velocidade de emergência de plântulas em areia, e 10, inferioridade dos lotes 4 e 7 , sendo os demais de comportamento intermediário (Tabela 1).

É interessante observar que lotes com bom desempenho no teste de germinação podem apresentar comportamento distinto quando expostos a situações adversas, como os lotes 5 e 9, que tiveram 97 e 96\% de germinação, respectivamente, na primeira época, porém, quando expostos a condições 
Tabela 1 - Resultados do grau de umidade (GU), geminação (G), primeira contagem de germinação (PCG), envelhecimento acelerado (EA), emergência de plântula em campo (EPC), emergência de plântula em areia (EPA) e velocidade de emergência de plântulas em areia (VEA) e de análise de imagens, por meio do comprimento de plântulas (CP) e índices de vigor (IV), de crescimento de plântulas (IC), uniformidade de crescimento (IUC), de dez lotes de sementes de girassol, cultivar Catissol 01, primeira e segunda época experimental.

\begin{tabular}{|c|c|c|c|c|c|c|c|c|c|c|c|}
\hline LOTE & GU & G & PCG & EA & EPC & EPA & VEA & $\mathrm{CP}$ & IV & IC & IUC \\
\hline \multicolumn{12}{|c|}{ 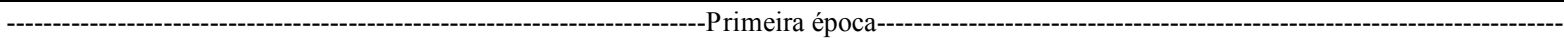 } \\
\hline 1 & 9,8 & $90 b^{*}$ & $82 \mathrm{~b}$ & $46 \mathrm{c}$ & $84 \mathrm{a}$ & $91 \mathrm{~b}$ & $11 \mathrm{~b}$ & $8,8 \mathrm{c}$ & $809 \mathrm{c}$ & $788 \mathrm{c}$ & $830 \mathrm{~b}$ \\
\hline 2 & 10,2 & $85 \mathrm{c}$ & $83 \mathrm{~b}$ & $60 \mathrm{~b}$ & $82 \mathrm{~b}$ & $84 \mathrm{~b}$ & $11 \mathrm{~b}$ & $8,2 \mathrm{c}$ & $788 \mathrm{c}$ & $751 \mathrm{c}$ & $826 \mathrm{~b}$ \\
\hline 3 & 10,5 & $90 \mathrm{~b}$ & $86 \mathrm{~b}$ & $73 a$ & $90 \mathrm{a}$ & $95 \mathrm{a}$ & $12 \mathrm{a}$ & $10,4 \mathrm{~b}$ & $874 \mathrm{~b}$ & $866 \mathrm{~b}$ & $882 \mathrm{a}$ \\
\hline 4 & 9,5 & $76 \mathrm{~d}$ & $63 \mathrm{~d}$ & $45 \mathrm{c}$ & $55 \mathrm{~d}$ & $56 \mathrm{~d}$ & $8 \mathrm{c}$ & $6,8 \mathrm{~d}$ & $718 \mathrm{~d}$ & $632 \mathrm{~d}$ & $804 \mathrm{~b}$ \\
\hline 5 & 10,4 & $97 \mathrm{a}$ & $82 \mathrm{~b}$ & $53 \mathrm{c}$ & 89 a & $93 \mathrm{a}$ & $12 \mathrm{a}$ & $11,6 \mathrm{a}$ & $919 \mathrm{a}$ & $964 \mathrm{a}$ & $875 \mathrm{a}$ \\
\hline 6 & 9,7 & $88 \mathrm{~b}$ & $68 \mathrm{~d}$ & $52 \mathrm{c}$ & $85 \mathrm{a}$ & $87 \mathrm{~b}$ & $10 \mathrm{~b}$ & $9 \mathrm{c}$ & $816 \mathrm{c}$ & $754 \mathrm{c}$ & $879 a$ \\
\hline 7 & 9,6 & $76 \mathrm{~d}$ & $73 \mathrm{c}$ & $61 \mathrm{~b}$ & $64 c$ & $64 \mathrm{c}$ & $9 \mathrm{c}$ & $7,7 \mathrm{~d}$ & $761 \mathrm{~d}$ & $715 \mathrm{c}$ & $808 \mathrm{~b}$ \\
\hline 8 & 10,1 & $85 \mathrm{c}$ & $75 \mathrm{c}$ & $64 \mathrm{~b}$ & $85 \mathrm{a}$ & $79 \mathrm{~b}$ & $11 \mathrm{~b}$ & $8,2 \mathrm{~b}$ & $851 \mathrm{~b}$ & $854 \mathrm{~b}$ & $847 \mathrm{~b}$ \\
\hline 9 & 10,6 & $96 a$ & $91 \mathrm{a}$ & $47 \mathrm{c}$ & $93 \mathrm{a}$ & $97 \mathrm{a}$ & $11 \mathrm{~b}$ & $11,5 \mathrm{a}$ & $933 \mathrm{a}$ & $968 \mathrm{a}$ & 897 a \\
\hline 10 & 10,2 & $99 \mathrm{a}$ & $97 \mathrm{a}$ & $75 \mathrm{a}$ & $94 \mathrm{a}$ & $94 \mathrm{a}$ & $12 \mathrm{a}$ & $11,8 \mathrm{a}$ & 896 a & $955 \mathrm{a}$ & $904 \mathrm{a}$ \\
\hline CV (\%) & - & 5,7 & 5,9 & 15 & 5,6 & 5,3 & 5,3 & 8,7 & 4,4 & 8,4 & 3,3 \\
\hline & & & & & -Segund & poca--- & - & & & - & -------- \\
\hline 1 & 7,8 & $80 \mathrm{a}$ & $78 \mathrm{~b}$ & $47 \mathrm{c}$ & $64 \mathrm{~b}$ & $66 \mathrm{~b}$ & $8,3 \mathrm{~b}$ & $7,5 \mathrm{c}$ & $749 \mathrm{c}$ & $654 \mathrm{~b}$ & $842 \mathrm{~b}$ \\
\hline 2 & 7,6 & $81 \mathrm{~b}$ & $79 \mathrm{~b}$ & $60 \mathrm{a}$ & $73 \mathrm{~b}$ & $75 \mathrm{~b}$ & $9,1 \mathrm{a}$ & $7,8 \mathrm{c}$ & $766 \mathrm{c}$ & $693 \mathrm{~b}$ & $848 \mathrm{~b}$ \\
\hline 3 & 7,7 & $86 \mathrm{~b}$ & $84 \mathrm{~b}$ & $66 \mathrm{a}$ & $82 \mathrm{a}$ & $84 \mathrm{a}$ & $11,3 \mathrm{a}$ & $10 \mathrm{a}$ & $860 \mathrm{a}$ & $835 \mathrm{a}$ & $894 \mathrm{a}$ \\
\hline 4 & 7,4 & $61 \mathrm{~d}$ & $58 \mathrm{~d}$ & $42 \mathrm{c}$ & $60 \mathrm{~b}$ & $56 \mathrm{~b}$ & $4,4 \mathrm{~d}$ & $5,4 \mathrm{~d}$ & $651 \mathrm{~d}$ & $501 \mathrm{c}$ & $799 \mathrm{c}$ \\
\hline 5 & 7,7 & $80 \mathrm{~b}$ & $78 \mathrm{~b}$ & $67 \mathrm{~b}$ & $79 \mathrm{a}$ & $83 \mathrm{a}$ & $9,5 \mathrm{a}$ & $9,1 \mathrm{~b}$ & $821 \mathrm{~b}$ & 793 a & $849 b$ \\
\hline 6 & 7,7 & $85 \mathrm{~b}$ & $83 \mathrm{~b}$ & $68 \mathrm{~b}$ & $66 \mathrm{~b}$ & $83 \mathrm{a}$ & $9,4 \mathrm{a}$ & $7,5 \mathrm{c}$ & $757 \mathrm{c}$ & $665 \mathrm{~b}$ & 848 b \\
\hline 7 & 7,9 & $75 \mathrm{c}$ & $71 \mathrm{c}$ & $46 \mathrm{c}$ & $62 \mathrm{~b}$ & $73 \mathrm{~b}$ & $6,4 \mathrm{c}$ & $4,9 \mathrm{~d}$ & $629 \mathrm{~d}$ & $483 \mathrm{c}$ & $775 \mathrm{c}$ \\
\hline 8 & 7,8 & $75 \mathrm{c}$ & $76 \mathrm{~b}$ & $66 a$ & $69 \mathrm{~b}$ & $70 \mathrm{~b}$ & $10,2 \mathrm{a}$ & $8,6 \mathrm{~b}$ & $800 \mathrm{~b}$ & $738 \mathrm{~b}$ & $862 \mathrm{~b}$ \\
\hline 9 & 7,9 & $90 \mathrm{a}$ & $88 \mathrm{a}$ & $63 \mathrm{a}$ & $79 \mathrm{a}$ & $81 \mathrm{a}$ & $11,4 \mathrm{a}$ & $8,8 \mathrm{~b}$ & $811 \mathrm{~b}$ & $794 \mathrm{a}$ & $864 \mathrm{~b}$ \\
\hline 10 & 8,1 & $96 \mathrm{a}$ & $94 \mathrm{a}$ & $75 \mathrm{a}$ & $85 \mathrm{a}$ & $88 \mathrm{a}$ & $10,4 \mathrm{a}$ & $10,1 \mathrm{a}$ & $870 \mathrm{a}$ & $845 \mathrm{a}$ & $894 \mathrm{a}$ \\
\hline CV (\%) & - & 6,3 & 6,5 & 12,2 & 5,5 & 12,5 & 11,9 & 10,4 & 4,9 & 8,6 & 3,2 \\
\hline
\end{tabular}

*Médias seguidas pela mesma letra, nas colunas, não diferem entre si pelo Teste de Scott Knott a 5\% de probabilidade.

de alta temperatura e umidade no envelhecimento acelerado, originaram somente 53 e $47 \%$ de plântulas normais, respectivamente, estando entre os lotes de pior desempenho na referida avaliação (Tabela 1). ALBUQUERQUE et al. (2001) e BRAZ \& ROSSETTO (2009) também tiveram sucesso na diferenciação dos lotes em diferentes níveis de vigor com o uso do teste de envelhecimento acelerado para sementes de girassol.

No teste de emergência de plântulas em campo e em areia, foi possível observar menor desempenho dos lotes 4, 5 e 7, comparado aos resultados de germinação, evidenciando claramente o efeito do ambiente no vigor das sementes. Esse teste ratifica, complementa e auxilia na definição do potencial fisiológico, pois estima o desempenho de sementes e lotes em condições variadas de ambiente (MENEZES et al., 2007). Sendo assim, o uso de testes baseados em diferentes princípios pode contribuir para obtenção de respostas mais seguras a respeito do potencial fisiológico de lotes de sementes.

A análise de imagens de plântulas (Figura 1) possibilitou a identificação de diferenças de potencial fisiológico entre lotes por meio de todos os parâmetros avaliados (Tabela 1), contudo, o comprimento de plântula e os índices de vigor e de crescimento de plântulas possibilitaram a separação dos lotes em três níveis de vigor, assim como os testes tradicionais, indicando maior desempenho dos lotes 5, 9 e 10, pior desempenho dos lotes 4 e 7, sendo os demais de comportamento intermediário. Resultados semelhantes foram verificados para sementes de várias espécies, os quais encontraram associação entre a classificação de lotes quanto ao vigor com a análise de imagens de plântulas com o sistema SVIS ${ }^{\circledR}$ e com a emergência de plântulas para sementes de melão (MARCOS FILHO et al., 2006) e de berinjela (SILVA \& CICERO, 2013), envelhecimento acelerado para 


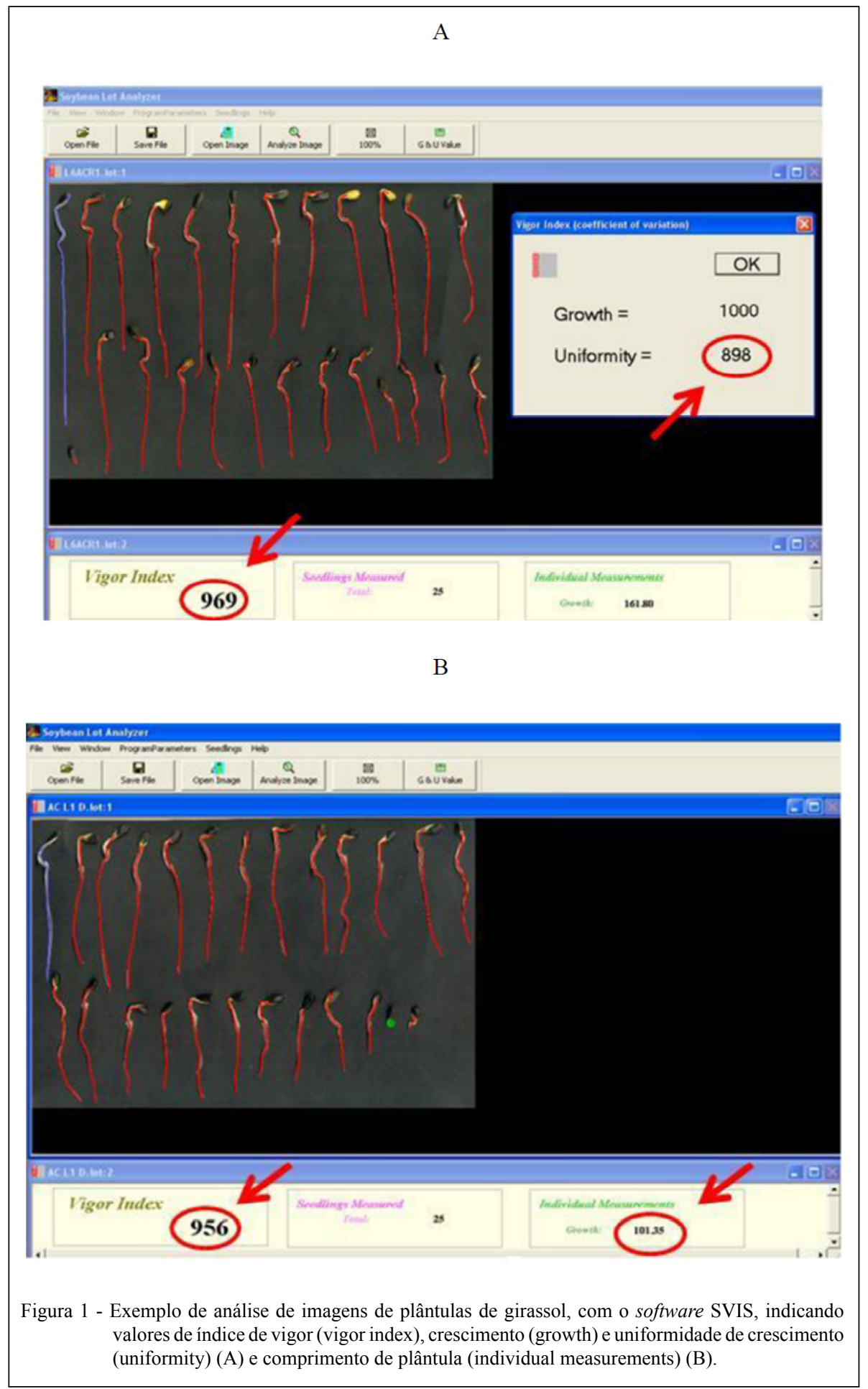

soja (MARCOS FILHO et al., 2009) e milho doce (ALVARENGA et al., 2012) e primeira contagem de germinação para sementes de alface (CONTRERAS \& BARROS, 2005).
Assim como na primeira época experimental, na segunda época, aos quatro meses de armazenamento, foi possível identificar diferenças entre os lotes com todos os testes

Ciência Rural, v.45, n.6, jun, 2015. 
utilizados (Tabela 1), havendo novamente a formação de três grupos quanto ao nível de vigor, com destaque para maior desempenho dos lotes 9 e 10, baixo desempenho dos lotes 4 e 7 e comportamento intermediário dos demais, de forma geral. Esses resultados assemelham-se com aqueles obtidos na caracterização inicial.

A análise de imagens de plântulas revelou resultados similares àqueles dos demais testes, apontando maior vigor do lote 10 , menor vigor dos lotes 4 e 7 e vigor médio para os demais (Tabela 1). É interessante observar, por exemplo, que os lotes 4 e 7 obtiveram baixo desempenho quanto a primeira contagem de germinação, velocidade de emergência de plântulas e envelhecimento acelerado, assim como menor comprimento de plântulas e menores índices de vigor, crescimento e uniformidade de crescimento. Outro resultado interessante é que o lote 7 obteve a mesma classificação de desempenho do lote 8 no teste de germinação, mas teve baixo desempenho em todas as avaliações de vigor, o que reforça a premissa de que o potencial de lotes deve ser avaliado por mais de um teste, os quais baseiam-se em princípios diferentes, possibilitando assim uma informação mais completa sobre o potencial de desempenho dos lotes.

Possivelmente, as sementes dos lotes 4 e 7 estavam em estádio mais avançado de deterioração, comparadas às dos demais lotes, o que resultou em maior demora na reestruturação do sistema de membranas celulares, refletindo no metabolismo e eficiência das mitocôndrias, com menor liberação de energia e maior lentidão de germinação e crescimento de plântulas.

Diante do exposto, ficou evidenciada a possibilidade da inclusão de análise computadorizada de imagens de plântulas na avaliação da qualidade de sementes de girassol, pois, além da coerência dos resultados com os obtidos nos testes tradicionalmente utilizados, o uso deste sistema pode trazer vantagens, como rapidez na obtenção dos resultados, maior confiabilidade e precisão e menor subjetividade da análise, pois evitam-se falhas por diferenças de critérios de avaliação utilizados pelos analistas de diferentes laboratórios (MARCOS FILHO et al., 2009). Além disso, deve-se considerar que testes que utilizam alta velocidade de captura de imagens e de processamento de dados são os mais indicados para fornecer alto nível de precisão nas análises de qualidade de sementes (DELL'ÁQUILA, 2007); seu uso também possui vantagens adicionais como proporcionar o arquivamento de imagens para análise posterior e, além disso, é um sistema de baixo custo, equivalente ao do teste de germinação (GOMES JUNIOR et al., 2009).

\section{CONCLUSÃO}

A análise computadorizada de imagens de plântulas é eficiente para avaliação do vigor de sementes de girassol.

\section{AGRADECIMENTOS}

Ao Conselho Nacional de Desenvolvimento Científico e Tecnológico $(\mathrm{CNPq})$, pela concessão das bolsas de estudo e recursos para condução da pesquisa, e à empresa Piraí Sementes, pela cessão das sementes.

\section{REFERÊNCIAS}

ALBUQUERQUE, M.C.F.; CARVALHO, N.M. Effects of the environmental stress on the emergence of sunflower, soybean and maize seeds with different levels of vigor. Seed Science and Technology, v.31, n.2, p.465-479, 2003. Disponível em: <http://www.ingentaconnect.com/content/ista/ sst/2003/00000031/00000002/art00023?crawler=true>. Acesso em: janeiro de 2014 .

ALVARENGA, R. et al. Avaliação do vigor de sementes de milho super doce por meio da análise computadorizada de imagens de plântulas. Revista Brasileira de Sementes, v. 34, n.3 3, p. 488 - 494, 2012. Disponível em: $<$ http://www.scielo.br/scielo.php?script=sci_arttext\&pi $\mathrm{d}=\mathrm{S} 0101-31222012000300017>$. Acesso em: outubro de 2013.

BAALBAKI, R. et al. Seed vigor testing handbook. Ithaca: Association of official seed analysts, 2009. 341p. (Contribuition, 32).

BRASIL. Ministério da Agricultura, Pecuária e Abastecimento. Regras para análise de sementes. Brasília, 2009. 395p.

BRAZ, M.R.S.; ROSSETTO, C.A.V. Correlação entre testes para avaliação da qualidade de sementes de girassol e emergência das plântulas em campo. Ciência Rural, v.39, n.7, p.2004-2009, 2009. Disponível em: <http:/ www.scielo.br/scielo.php?pid=S0103-84782009000700008\&script $=$ sci arttext $>$. Acesso em: dezembro de 2013.

CHIQUITO, A.A. et al. Assessment of physiological potential of cucumber seeds using the software Seedling Vigor Imaging System ${ }^{\circledR}$. Revista Brasileira de Sementes, v.34, n.2 p.255-263, 2012. Disponível em: <http://www.scielo.br/scielo.php?script=sci_arttext\& pid=S0101-31222012000200010 $>$. Acesso em: janeiro de 2014 .

CONTRERAS, S.; BARROS, M.Vigor tests on lettuce seeds and their correlation with emergence. Ciencia e Investigacion Agraria, v.32, n.1, p.3-10, 2005.

DELL'AQUILA, A. Pepper seed germination assessed by combined X-radiography and computer-aided imaging analysis. Biologia Plantarum, v.51, p.777-781, 2007.Disponível em: $<$ http://link.springer.com/article/10.1007/s10535-007-0159-9>. Acesso em: dezembro de 2013.

FAGUNDES, M.H. Sementes de girassol: alguns comentários. Brasília: CONAB, 2009. Disponível em: <http://www.conab.gov. br/download/cas/especiais/Semente-de-Girassol.pdf $>$. Acesso em: novembro de 2012.

GOMES JUNIOR, F.G. et al. Evaluation of priming effects on sweet corn seeds by SVIS. Seed Technology, v.31, n.1, p.95-100, 2009. 
MAGUIRE, J.D. Speed of germination-aid in relation evaluation for seedling emergence vigor. Crop Science, v.2, n.2, p.176-177, 1962.

MENEZES, N.L. et al. Teste de condutividade elétrica em sementes de aveia preta. Revista Brasileira de Sementes, v.29, n.2, p.138-142, 2007. Disponível em: <http://www.scielo.br/ scielo.php?script=sci_arttext\&pid=S0101-31222007000200019>. Acesso em: novembro de 2013.

MARCOS FILHO, J. Teste de envelhecimento acelerado. In: KRZYZANOWSKI, F.C. (Ed.). Vigor de sementes: conceitos e testes. Londrina: ABRATES, 1999. p.1-24.

MARCOS FILHO, J. et al. Assessment of melon seed vigour by an automated computer imaging system compared to traditional procedures. Seed Science and Technology, v.34, n.2, p.507519, 2006. Disponível em: <http://www.ingentaconnect.com/ content/ista/sst/2006/00000034/00000002/art00023>. Acesso em: novembro de 2013

MARCOS FILHO, J. et al. Métodos para avaliação do vigor de sementes de soja, incluindo a análise computadorizada de imagens. Revista Brasileira de Sementes, v.31, n.1, p.102112, 2009. Disponível em: <http://www.scielo.br/scielo. php? script $=$ sci_arttext\&pid $=$ S0101-31222009000100012>. Acesso em: outubro de 2013.
OHLSON, O.C. et al. Teste de envelhecimento acelerado em sementes de trigo. Revista Brasileira de Sementes, v.32, n.4, p.118-124, 2010. Disponível em: <http://www.scielo.br/scielo.php?pid=S010131222010000400013\&script=sci_arttext>. Acesso em: janeiro de 2014.

SILVA, V.N.; CICERO, S.M. Sistema de análise de imagens de plântulas para avaliação do potencial fisiológico de sementes de berinjela. Hoticultura Brasileira, v.32, n.2, p. 145-15 2014.

SILVA, V.N. et al. Computerized imaging analysis of seedlings for assessment of physiological potential of wheat seeds. Revista Brasileira de Sementes, v.34, n.4, p.589-596, 2012a. Disponível em: <http://www. scielo.br/scielo.php?pid=S0101-31222012000400009\&script $=$ sci_ arttext. Acesso em: novembro de 2013.

SILVA, C.B. et al. Automated system of seedling image analysis (SVIS) and electrical conductivity to assess sun hemp seed vigor. Revista Brasileira de Sementes, v.34, n.1 p.55-60, 2012b. Disponível em: <http://www.scielo.br/scielo.php?script=sci_arttext \&pid=S0101-31222012000100007>. Acesso em: outubro de 2013.

TEKRONY, D.M. Precision is an essential component in seed vigour testing. Seed Science and Technology, v.31, n.3, p.435-447, 2003. Disponível em: <http://www.ingentaconnect.com/content/ ista/sst $/ 2003 / 00000031 / 00000002 /$ art00020? $\mathrm{crawler}=$ true $>$. Acesso em: setembro de 2013. 